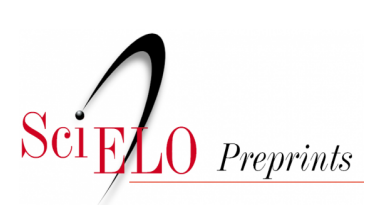

Situação: O preprint não foi submetido para publicação

\title{
Fluxo de internação por COVID-19 nas regiões de saúde do Brasil
}

Everton Nunes da Silva, Fernando Ramalho Gameleira Soares, Gustavo Saraiva Frio, Aimê Oliveira, Fabrício Vieira Cavalcante, Natália Regina Alves Vaz Matins, Klébya Hellen Dantas Oliveira, Claudia Cristina de Aguiar Pereira, Ivana Cristina de Holanda Cunha Barreto, Mauro Niskier Sanchez, Fernando José Herkrath, Leonor Maria Pacheco Santos

https://doi.org/10.1590/SciELOPreprints.1849

Este preprint foi submetido sob as seguintes condições:

- O autor submissor declara que todos os autores responsáveis pela elaboração do manuscrito concordam com este depósito.

- Os autores declaram que estão cientes que são os únicos responsáveis pelo conteúdo do preprint e que o depósito no SciELO Preprints não significa nenhum compromisso de parte do SciELO, exceto sua preservação e disseminação.

- Os autores declaram que a pesquisa que deu origem ao manuscrito seguiu as boas práticas éticas e que as necessárias aprovações de comitês de ética de pesquisa estão descritas no manuscrito, quando aplicável.

- Os autores declaram que os necessários Termos de Consentimento Livre e Esclarecido de participantes ou pacientes na pesquisa foram obtidos e estão descritos no manuscrito, quando aplicável.

- Os autores declaram que a elaboração do manuscrito seguiu as normas éticas de comunicação científica.

- Os autores declaram que o manuscrito não foi depositado e/ou disponibilizado previamente em outro servidor de preprints ou publicado em um periódico.

- O autor submissor declara que as contribuições de todos os autores estão incluídas no manuscrito.

- O manuscrito depositado está no formato PDF.

- Os autores declaram que caso o manuscrito venha a ser postado no servidor SciELO Preprints, o mesmo estará disponível sob licença Creative Commons CC-BY.

- Caso o manuscrito esteja em processo de avaliação ou sendo preparado para publicação mas ainda não publicado por um periódico, os autores declaram que receberam autorização do periódico para realizar este depósito.

Submetido em (AAAA-MM-DD): 2021-02-13

Postado em (AAAA-MM-DD): 2021-02-18 


\section{Título completo}

Fluxo de internação por COVID-19 nas regiões de saúde do Brasil

Inpatient flow for COVID-19 in the Brazilian health regions

\section{Autores:}

Everton Nunes da Silva ${ }^{1,2}$ (ORCID ID 0000-0001-8747-4185), evertonsilva@unb.br

Fernando Ramalho Gameleira Soares ${ }^{3}$ (ORCID 0000-0001-8244-1800) fernandorgsoares@gmail.com

Gustavo Saraiva Frio² (ORCID 0000-0001-6453-312X), gustavo.frio@gmail.com

Aimê Oliveira² (ORCID 0000-0002-3084-6491), oliveira.aime@gmail.com

Fabrício Vieira Cavalcante ${ }^{2}$ (ORCID 0000-0002-8706-0457), fabricioocavalcante@gmail.com

Natália Regina Alves Vaz Martins² (ORCID 0000-0003-1162-8795) natalia.ravm@gmail.com

Klébya Hellen Dantas de Oliveira² (ORCID 0000-0002-3600-4009), oliveirakhd@gmail.com

Claudia Cristina de Aguiar Pereira ${ }^{4}$ (ORCID: 0000-0003-1389-9214), pereirac.claudia@gmail.com

Ivana Cristina de Holanda Cunha Barreto ${ }^{5}$ (ORCID: 0000-0001-8447-3654), ivana.barreto24@gmail.com

Mauro Niskier Sanchez² (ORCID ID 0000-0002-0472-1804), mauro.sanchez4@gmail.com

Fernando José Herkrath ${ }^{6}$ (ORCID: 0000-0003-4439-0189), fernandoherkrath@gmail.com

Leonor Maria Pacheco Santos² (ORCID 0000-0002-6739-6260), leopac@unb.br

${ }^{1}$ Curso de Saúde Coletiva, Faculdade de Ceilândia, Universidade de Brasília, Brasil.

2 Programa de Pós-Graduação em Saúde Coletiva, Faculdade de Ciências da Saúde, Universidade de Brasília, Brasil.

${ }^{3}$ Programa de Pós-Graduação em Saúde Pública, Escola Nacional de Saúde Pública Sérgio Arouca, Fundação Oswaldo Cruz, Rio de Janeiro, Brasil.

${ }^{4}$ Fundação Oswaldo Cruz, Escola Nacional de Saúde Pública, Rio de Janeiro, Brasil.

${ }^{5}$ Fundação Oswaldo Cruz, Ceará, Brasil.

${ }^{6}$ Instituto Leônidas e Maria Deane, Fundação Oswaldo Cruz, Manaus, Brasil 


\section{Autor de correspondência:}

Everton Nunes da Silva, evertonsilva@unb.br

Campus Universitário, s/n, Centro Metropolitano, Brasília - DF, 72220-275, telefone:(61) 3107-8418

Contribuições dos Autores: ENS, participou da pesquisa, contribuiu na metodologia, na coordenação da análise de dados da elaboração do manuscrito e da revisão do texto final; FRGS e GSF participou da pesquisa, contribuiu na metodologia, na análise de dados, elaboração do manuscrito e da revisão do texto final; AO, FVC, NRAVM, KHDO,CCAO, ICHCB, MNS, FJH participaram da pesquisa, contribuíram na metodologia, na escrita do rascunho e na revisão do texto final; SANTOS LMP concebeu a pesquisa, contribuiu na metodologia, na interpretação dos resultados, na escrita do rascunho e na revisão do texto final.

Agradecimentos: Agradecemos aos Ministérios da Ciência e Tecnologia e Ministério da Saúde, que por meio da Chamada Pública MCTI/CNPq/CTSaúde/MS/SCTIE/Decit № 07/2020 financiou o presente trabalho.

Conflitos de Interesse: Os autores declaram ausência de conflitos de interesse. 


\section{Resumo}

Objetivo: Investigar os fluxos de internações por COVID-19 nas 450 regiões e 117 macrorregiões de saúde brasileiras no período de março a outubro de 2020. Método: Estudo descritivo, compreendendo todas as internações por COVID-19 registradas no Sistema de Informação de Vigilância Epidemiológica da Gripe (SIVEP-Gripe) entre a $8^{\underline{a}}$ e a $44^{\underline{a}}$ semanas epidemiológicas de 2020. Foi calculada a proporção das internações por COVID-19 realizadas pelos residentes que ocorreram dentro da sua respectiva região de saúde, estratificado segundo períodos de maior e menor demanda de internações e segundo o porte populacional das regiões de saúde. Foi calculado o indicador de eficácia migratória, que leva em consideração a evasão e invasão de pacientes, por meio do cruzamento dos dados de origem dos pacientes (região de saúde de residência) com os dados do local da realização das internações (região de saúde de atendimento). Resultados: Foram identificadas 397.830 internações por COVID-19 no período. A evasão foi de $11,9 \%$ dos residentes nas regiões de saúde e de 6,8\% nas macrorregiões; o padrão que se manteve também no período de pico das internações por COVID-19. Houve em média $17,6 \%$ de evasão dos residentes das regiões de saúde do Nordeste e de $8,8 \%$ nas regiões de saúde do Sul. A evasão foi mais acentuada nas regiões de saúde com até $100 \mathrm{mil} / \mathrm{hab}$. (36,9\%), a qual foi 7 vezes maior que a verificada nas regiões de saúde com mais de 2 milhões/habitantes (5,2\%). O indicador de eficácia migratória negativo $(-0,39)$ indicou predomínio da evasão. Das 450 regiões de saúde brasileiras, $117(39,3 \%)$ apresentaram coeficiente de eficácia migratória entre -1 e $-0,75$ e $113(25,1 \%)$ entre $-0,75$ e - $-0,25$. Conclusão: Os resultados indicam que a regionalização do sistema de saúde mostrou-se adequada na organização do atendimento no território, porém as longas distâncias percorridas ainda são preocupantes.

Palavras-chave: COVID-19; Regionalização; Hospitalização; Transferência de Pacientes. 


\section{Summary}

Objective: To investigate the flows of hospitalizations for COVID-19 in the 450 regions and 117 Brazilian health macro-regions between March and October 2020. Method: Descriptive study, comprising all hospitalizations due to COVID19 registered in the Flu Epidemiological Surveillance Information System (SIVEPGripe) between the 8th and 44th epidemiological weeks of 2020. The proportion of hospitalizations for COVID-19 occurred within same health region of residency was calculated, stratified according to periods of greater and lesser demand for health care, according to the population size of health regions. The indicator of migratory efficacy was calculated, which takes into account the evasion and invasion of patients, by crossing the data of origin of the patients (health region of residence) with the data of the place of hospitalization (health region of attendance). Results: 397,830 admissions were identified for COVID-19 in the period. Evasion was $11.9 \%$ of residents in health regions and $6.8 \%$ in macroregions, pattern that was maintained during the peak period of hospitalizations for COVID-19. There was an average of $17.6 \%$ of evasion of residents of health regions in the Northeast and of $8.8 \%$ in health regions of the South. Evasion was more accentuated in health regions with up to 100 thousand / inhabitants (36.9\%), which was 7 times greater than that observed in health regions with more than 2 million / inhabitants $(5.2 \%)$. The negative migratory efficacy indicator $(-0.39)$ indicated a predominance of evasion. Of the 450 Brazilian health regions, 117 (39.3\%) had a coefficient of migratory efficacy between -1 and -0.75 and 113 (25.1\%) between -0.75 and -0.25 . Conclusion: The results indicate that the regionalization of the health system proved to be adequate in the organization of care in the territory, however the long distances traveled are still worrying.

Keywords: COVID-19; Regionalization; Hospitalization; Patient Transfer. 


\section{Introdução}

A COVID-19 tem desafiado os sistemas de saúde mundialmente. Sua alta transmissibilidade, potencialmente agravada pelo aparecimento de novas variantes, e ausência de tratamentos específicos têm forçado os países a adotar medidas de isolamento ou distanciamento social (1-4). Mesmo com o uso de vacinas contra a COVID-19, as medidas de prevenção devem ser mantidas devido à produção global insuficiente dos imunizantes (5). Essas medidas têm sido tomadas com o intuito de reduzir o número de pacientes com sintomas moderados e graves, os quais requerem cuidados hospitalares, seja em leitos de enfermaria, seja em unidades de tratamento intensivo (UTI). Além da questão de saúde da população, busca-se evitar o colapso do sistema de saúde, visto a forte pressão da COVID-19 nos sistemas e serviços de saúde, dependendo da velocidade de propagação do vírus SARS-CoV-2, causador da COVID-19 (6).

No Brasil, a transmissão do vírus SARS-CoV-2 se fez presente de maneira rápida em todas as regiões brasileiras, em diferentes proporções $(7,8)$. Até a semana epidemiológica 44 (26/10/2020), foram hospitalizados 466.815 casos de COVID-19 segundo registros do Sistema de Informação da Vigilância Epidemiológica da Gripe (SIVEP-Gripe) (9).

O processo de interiorização da doença, ou seja, a disseminação para municípios de pequeno porte, o que coloca em xeque a capacidade de gestão municipal (10). Um estudo apontou que 90,4\% dos municípios brasileiros não dispunham de leitos de UTI para adultos, 59,3\% não dispunham de respiradores/ ventiladores, 51,9\% não possuíam monitores de eletrocardiograma (ECG), $39,6 \%$ não contavam com desfibriladores, $71,0 \%$ não registravam bombas de infusão e $84,6 \%$ não tinham tomógrafos registrados no Cadastro Nacional de Estabelecimentos de Saúde (CNES) em fevereiro de 2020 (10).

Nesse contexto, é importante analisar os fluxos de internações por COVID-19 no âmbito das regiões de saúde, estabelecidas como uma estratégia para otimizar a gestão dos serviços de saúde, a racionalização de recursos e o aporte institucional para a criação de redes de atenção em saúde (11). A organização regionalizada dos serviços de saúde no Sistema Único de Saúde (SUS) define os fluxos de pacientes no território, tanto no nível das regiões como no nível das 
macrorregiões de saúde, e depende, entre outros, da oferta de transporte e da capacidade dos municípios de referência $(12,13)$.

O objetivo deste estudo foi de investigar os fluxos de internações por COVID-19 nas 450 regiões e 117 macrorregiões de saúde brasileiras no período de março a outubro de 2020. Avaliou-se: i) a proporção de internações de residentes dentro da sua região de saúde e da sua macrorregião de saúde, estratificado pelo período de maior estresse do sistema de saúde; ii) fluxos de pacientes que buscaram internações por COVID-19 entre as regiões de saúde, mapeando as regiões e macrorregiões que apresentaram processo migratório de evasão/ invasão de internações por COVID-19.

\section{Métodos}

\section{Desenho do estudo}

Trata-se de um estudo descritivo que busca investigar os fluxos de internações por COVID-19 nas regiões de saúde, levando em consideração diferentes recortes. A unidade de análise foi a região de saúde, contemplando todas as 450 regiões e as 117 macrorregiões de saúde brasileiras. O estudo compreendeu todas as internações por COVID-19 registradas no SIVEP-Gripe de $1^{\circ}$ de março a 29 de outubro de 2020. Os dados compreendem notificações entre a $8^{\underline{a}}$ e a $44^{\underline{a}}$ semanas epidemiológicas. Este período foi selecionado devido à disponibilidade de dados até a realização deste estudo.

\section{Variáveis e fontes de dados}

Para estimar a capacidade de atendimento das regiões de saúde em prover internações por COVID-19 aos seus residentes, foi calculada a proporção obtida pela divisão do somatório de todas as internações por COVID-19 realizadas pelos residentes dentro da sua respectiva região de saúde pelo somatório de todas as internações por COVID-19 realizadas pelos residentes daquela região de saúde. Esta proporção foi calculada em dois períodos distintos. O primeiro período refere-se ao pico das internações por COVID-19 na região de saúde, definido como a semana epidemiológica com maior número de internações por COVID-19, acrescida das semanas epidemiológicas anterior e posterior. Este 
período representa a situação em que o sistema hospitalar seria mais demandado pela população atendida. O segundo período refere-se às demais semanas epidemiológicas, excluindo-se o período de pico das internações por COVID-19.

Para identificar os fluxos de internações por COVID-19, foram cruzados os dados de origem dos pacientes (região de saúde de residência) com os dados do local da realização das internações (região de saúde de atendimento). Com o cruzamento das internações por COVID-19, também foi possível identificar o indicador de eficácia migratória, que leva em consideração a evasão e invasão de pacientes. Segundo Rocha (14), quanto maior a evasão de pacientes, menor seria a capacidade de assistir local ou regionalmente a população; e quanto maior a invasão de pacientes, maior o poder de atração exercido pelas condições de oferta de assistência (14). O indicador de eficácia migratória (IEM) foi obtido pela fórmula abaixo:

$$
I E M=(I-E) /(E+I)
$$

Em que "I" é a invasão, definida como a entrada de pacientes de outras regiões de saúde; "E" é a evasão, definida com a saída de pacientes para outras regiões de saúde. "I - E" é a migração líquida (entradas menos saídas de pacientes em uma região de saúde). "E + l" é a migração bruta (entradas mais saídas de pacientes em uma região de saúde). O indicador assume valores entre 1 e -1, inclusive. Valores próximos de 1 indicam alta atração migratória, enquanto valores próximos de -1 indicam alta repulsão migratória.

Também foi calculada a distância média percorrida pelos pacientes para internações realizadas fora das regiões de saúde e entre municípios dentro da própria região. Esta foi calculada no software QGIS com base na distância euclidiana em metros entre as coordenadas das sedes dos municípios de residência e de internação do fluxo, utilizando uma projeção policônica com o sistema de referência geodésico SIRGAS 2000. As coordenadas das sedes municipais foram obtidas no estudo "Cadastro de Localidades Selecionadas 2010" do Instituto Brasileiro de Geografia e Estatística (IBGE). Esta informação fornece uma medida de trajeto percorrido pelos pacientes, a qual foi estratificada por porte populacional da região de saúde (até 100 mil habitantes; entre 100 mil 
e 200 mil habitantes; entre 200 mil e 500 mil habitantes; entre 500 mil e 1 milhão de habitantes; de 1 milhão a 2 milhões de habitantes; e acima de 2 milhões de habitantes) e região geográfica (Norte, Nordeste, Centro-Oeste, Sudeste e Sul).

O número de internações por COVID-19 foi obtido por meio do sistema SIVEPGripe do Ministério da Saúde do Brasil. Foram considerados para a amostra deste estudo apenas indivíduos nos quais a classificação final do caso foi de Síndrome Respiratória Aguda Grave (SRAG)-COVID-19, confirmada após o resultado positivo de teste molecular RT-PCR para o vírus SARS-CoV-2 (15).

\section{Análise estatística}

Os dados foram tabulados para cada uma das 450 regiões de saúde e das 117 macrorregiões de saúde. Inicialmente, foram apresentadas as frequências absolutas e relativas de internação por COVID-19. Em seguida, foram calculados os percentuais médios de evasão nas regiões de saúde para Brasil e suas cinco regiões geográficas, estratificando segundo períodos de maior e menor demanda de internações e segundo o porte populacional das regiões de saúde. O deslocamento foi representado pelas distâncias médias e graficamente por meio de mapas, incluindo os fluxos entre regiões e macrorregiões de saúde. Da mesma forma, a eficiência migratória nesses recortes territoriais foi apresentada por meio de mapas. Para a análise dos dados, foi utilizado o software STATA, versão 14. Para a elaboração dos mapas, foi utilizado o software QGIS, versão 3.12 .

\section{Aspectos éticos}

Foram utilizados exclusivamente dados secundários, de acesso público e não identificados. Dessa forma, não foi necessária a submissão do estudo ao sistema CEP/CONEP.

\section{Resultados}

No período de março a outubro de 2020, foram registradas no Brasil 397.830 internações por COVID-19, sendo 33.399 no Norte (8,4\%, taxa de 17,9 internações por 10.000 hab.), 81.121 no Nordeste (20,4\%, taxa de 14,1 
internações por 10.000 hab.), 37.626 no Centro-Oeste $(9,4 \%$, taxa de 22,8 internações por 10.000 hab.), 200.497 no Sudeste (50,4\%, taxa de 22,5 internações por 10.000 hab.) e 45.187 no Sul (11,4\%, taxa de 15 internações por 10.000 hab.).

Apenas 11,9\% dos residentes saíram de suas regiões de saúde para conseguir internação por COVID-19. Entretanto, estratificando a análise por regiões geográficas, o Nordeste destaca-se como a região com maior evasão de pacientes, onde se observou 17,6\%, o dobro do percentual verificado na Região Sul. Identificaram-se também variações em termos de porte populacional das regiões de saúde, com o percentual médio de evasão de 36,9\% naquelas regiões de saúde com população de até 100 mil habitantes e 5,2\% naquelas com população acima de 2 milhões de habitantes. Essa relação inversa entre porte populacional e percentual de evasão se mantém de forma geral, mesmo estratificando os dados pelas regiões geográficas brasileiras (Tabela 1).

As diferenças no percentual de evasão das regiões de saúde mostraram-se pouco sensíveis em relação aos períodos com maior ou menor demanda por internações, não sendo possível identificar um padrão evidente nas variações entre esses períodos quando avaliados por região geográfica e porte populacional. No entanto, as regiões geográficas apresentaram, com exceção do Sudeste, um pequeno incremento da evasão quando se analisou o período de maior demanda (Tabela 1).

As distâncias percorridas pelos pacientes nas 47.476 internações por COVID-19 registradas fora da região de saúde de residência somadas perfazem um total de 5,9 milhões de quilômetros, o que representa uma média de $229 \mathrm{~km}$ por deslocamento. Esse indicador, contudo, apresenta grande heterogeneidade, pois reúne deslocamentos médios que vão desde $60 \mathrm{~km}$ no Sudeste até $409 \mathrm{~km}$ no Norte. Embora o maior número de internações por COVID-19 tenha sido encontrado na região Sudeste $(45 \%)$ e os maiores deslocamentos médios na região Norte, seguido pela região Centro-Oeste $(182,8 \mathrm{~km})$, foi o Nordeste que apresentou o maior somatório de deslocamentos, mais de 1,9 milhão de km, uma vez que além de apresentar deslocamento médio relevante $(135,4 \mathrm{~km})$, teve o segundo maior volume de internações de residentes por COVID-19 (30\%). 
Os estados do Norte e do Centro-Oeste são aqueles que apresentaram maiores médias de deslocamento para fora das regiões de saúde (acima de $300 \mathrm{~km}$ ), sendo os principais, por ordem decrescente: Acre $(922,1 \mathrm{~km})$, Mato Grosso $(489,2$ km), Amazonas (484,2 km), Roraima (471,8 km), Mato Grosso do Sul (466,3km), Rondônia (459,9km), Pará (411km) e Amapá (315km) (Figura 1A).

De maneira geral, houve menor deslocamento médio para internação por COVID-19 dentro das regiões de saúde $(28,2 \mathrm{~km})$. Apenas alguns poucos estados apresentam médias de deslocamentos acima de $100 \mathrm{~km}$, são eles: Amazonas (134,4 km), Rondônia (122,9 km), Roraima (117,1 km), Acre (108,2 km) e Mato Grosso do Sul (103,7 km). É possível perceber na Figura 1B que o Sudeste, tanto em relação aos deslocamentos para fora das regiões de saúde, quanto entre os municípios dentro das regiões de saúde, apresentou menores deslocamentos médios. No deslocamento interno à região de saúde, registrouse no Sudeste uma média ligeiramente superior a $20 \mathrm{~km}$.

As Figuras 2A e 2B evidenciam os fluxos mais intensos de evasão de pacientes para internações por COVID-19 no Brasil, partindo das regiões e macrorregiões de saúde, entre março e outubro de 2020. É nítida a centralidade exercida pelas regiões de saúde dos municípios das capitais estaduais, figurando na maioria das vezes como a região de destino mais frequente dos fluxos de evasão para internação nas escalas das unidades da federação. Algumas regiões de saúde também extrapolam os limites estaduais, como é o caso da região de saúde do município de São Paulo, que exerce influência sobre todas as capitais brasileiras, sendo o destino mais frequente da evasão de muitas delas (Figura 2A). As capitais do Norte e Centro-Oeste centralizam fluxos de regiões que estão a grandes distâncias, como se observa mais nitidamente nos fluxos em direção às regiões de saúde de Manaus, Belém e Cuiabá.

Os fluxos entre macrorregiões de saúde, embora partam de um volume significativamente menor de deslocamentos (representando apenas 6,8\% das internações dos seus residentes), reforçam ainda mais a centralidade das capitais estaduais, e do movimento de várias capitais em direção à São Paulo quando os pacientes precisam se deslocar para alguma outra macrorregião (Figura 2B). 
Em contraste a esses grandes deslocamentos, verificou-se, em vários estados, a presença de polos de atração de fluxo de pacientes, além das capitais. Dentre eles, destacam-se principalmente aqueles relacionados às regiões de saúde dos municípios listados no Quadro 1. Pelo Índice de Desenvolviento Humano Municipal (IDHM) de 2010, a maioria dos municípios eram classificados com IDHM Muito Alto (12\%) e Alto (67\%), e apenas $21 \%$ tinham IDHM Médio, indicando que provavelmente são municípios mais desenvolvidos que os seus vizinhos. Analisando o porte populacional, comparado com os demais municípios nas suas respectivas macroregiões de saúde, $75 \%$ dos municípios eram os mais populosos da sua macroregião de saúde, atuando como polos para migração de pacientes (Quadro 1).

As regiões de saúde localizadas no Norte, especialmente em Manaus, Macapá e Belém, e nas capitais litorâneas de São Luiz, Fortaleza, Recife, Maceió, Rio de Janeiro, além do estado de São Paulo, atingiram o período crítico para internações por COVID-19 ainda em maio de 2020. Enquanto isso, regiões de saúde dos estados do Mato Grosso do Sul, Goiás e parte do interior da Bahia e Minas Gerais apresentaram picos mais tardios de internações por COVID-19. Em geral, as regiões de saúde distribuídas nas capitais do Sul tiveram seus momentos mais críticos de internação em julho (Figura 3).

Os dados de migração bruta para internação por COVID-19, considerando o deslocamento entre as macrorregiões, dão destaque em magnitude àquelas das Regiões Metropolitanas de São Paulo, Goiânia, Distrito Federal e entorno, Salvador, Recife e Fortaleza, todas com total de migração bruta acima de 800 deslocamentos. A região Sul, apesar de indicar maior eficácia migratória média entre as macrorregiões de saúde, apresenta baixa migração bruta entre elas. Grande parte das macrorregiões de saúde que tiveram uma quantidade maior de migração bruta de pacientes coincidem com áreas onde se verifica o predomínio de movimentos de invasão em relação à evasão, e se dão mais claramente nas capitais dos estados ou em importantes cidades do interior. Destacam-se movimentos migratórios com predomínio de evasão, mais relevantes em termos quantitativos, nas macrorregiões contíguas àquelas das capitais (Figura 4 A). 
O cálculo da eficácia migratória para as regiões de saúde resulta em um valor médio nacional de -0,39, o que indica que entre todas as regiões de saúde predominam processos de evasão. Vale ressaltar também que os deslocamentos entre regiões de saúde se dão ainda sobre uma base de migração bruta mais elevada do que a verificada entre as macrorregiões de saúde. A região Sul, mesmo figurando como a região geográfica que apresenta maior média de eficácia migratória de regiões de saúde para internação por COVID-19, ainda sim apresenta valor menor que zero para o indicador $(-0,17)$. As outras regiões geográficas mostram maior número de regiões de saúde com predomínio de evasão. A região Nordeste é a que apresenta a menor média do valor de eficácia migratória entre as regiões de saúde $(-0,52)$, seguida das regiões Centro-Oeste $(-0,46)$, Norte $(-0,45)$ e Sudeste $(-0,33)$ (Figura 4B).

\section{Discussão}

Os resultados do estudo revelaram um baixo percentual de evasão para internação por COVID-19 nas regiões de saúde brasileiras (11,9\%), conseguindo atender em média $88,1 \%$ dos seus residentes no período de março a outubro de 2020. Esse padrão se manteve também no período de pico das internações por COVID-19. No entanto, há variações substanciais quando os dados são estratificados por região geográfica e porte populacional. Houve em média $17,6 \%$ de evasão dos residentes das regiões de saúde do Nordeste, enquanto esse percentual foi de $8,8 \%$ nas regiões de saúde do Sul. A evasão foi mais acentuada nas regiões de saúde com até $100 \mathrm{mil} / \mathrm{hab}$. (36,9\%), a qual foi 7 vezes maior que a verificada nas regiões de saúde com mais de 2 milhões/hab. (5,2\%). Quando os residentes tiveram que procurar internações por COVID-19 em outras regiões de saúde, o deslocamento médio foi de $229 \mathrm{~km}$, sendo essa distância média menor no Sudeste $(60 \mathrm{~km})$ e maior no Norte (409km). O indicador de eficácia migratória negativo (-0,39), indicando predomínio da evasão, foi provavelmente influenciado pelas regiões de saúde menos populosas $(79,1 \%$ das regiões de saúde têm menos de $500 \mathrm{mil} / \mathrm{hab}$.), as quais são mais frequentes no território brasileiro. Das 450 regiões de saúde brasileiras, 117 (39,3\%) 
apresentaram coeficiente de eficácia migratória entre -1 e -0,75 e $113(25,1 \%)$ entre $-0,75$ e $-0,25$.

Quanto à resolutividade das redes de serviços nas regiões de saúde, outro estudo analisou os fluxos de internações em 2016. Os resultados apontaram para um percentual de evasão de $33 \%$ das internações realizadas no SUS, em um universo de mais de 11 milhões de internações (11). Salvas as diferenças metodológicas e do período analisado, nosso estudo revelou um percentual menor de evasão $(11,9 \%)$ para as internações por COVID-19.

A opção pelo recorte da região de saúde permitiu analisar a capacidade de cooperação entre os municípios de uma mesma região de saúde, dados os desafios enfrentados em nível municipal em termos de equipamentos e oferta de leitos (16). Historicamente, a descentralização do SUS para os entes subnacionais priorizou o movimento de municipalização da saúde até o ano de 2000, no qual os municípios ganhavam protagonismo na provisão e organização dos serviços de saúde (17). A baixa cooperação interfederativa e as escassas experiências de redes de atenção à saúde reforçaram a fragmentação do cuidado da saúde, comprometendo a implementação dos princípios da universalidade e da integralidade do SUS (18). A partir das Normas Operacionais de Assistência à Saúde (NOAS), publicadas em 2001 e 2002 (19), a regionalização da saúde ganhou espaço, bem como a organização das redes de atenção à saúde, que foram reguladas pela Portaria GM/MS nº 4.279/2010 (20). No ano seguinte, o Decreto ํㅜ 7.508/2011 (21) estabeleceu critérios mínimos de oferta de ações e serviços de saúde para ser instituídas as regiões de saúde, devendo conter pelo menos: i) atenção primária; ii) urgência e emergência; iii) atenção psicossocial; iv) atenção ambulatorial especializada e hospitalar; e v) vigilância em saúde. Todos esses cuidados têm uma interface direta com o enfrentamento da COVID-19 no contexto brasileiro.

Ademais, o Conselho Nacional de Secretarias Estaduais de Saúde (CONASS) e o Conselho Nacional de Secretárias Municipais de Saúde (CONASEMS) têm reforçado o papel da regionalização no seu guia orientador para o enfrentamento da pandemia de COVID-19 na rede de atenção à saúde (22). Entre as ações recomendadas com foco na regionalização da saúde, pode-se citar: i) a constituição de uma rede solidária para a aquisição de equipamentos de 
proteção individual (EPI), respiradores e outros insumos necessários; ii) a identificação de leitos de retaguarda já em funcionamento no âmbito da região de saúde; iii) o levantamento da ampliação de leitos conforme a necessidade de cada região de saúde.

É preciso compreender que o processo de regionalização da saúde no Brasil ainda está inacabado, e que a ausência de um ente legal instituído em nível regional no SUS, com arcabouço legal constituído, com poder para o planejamento e a operacionalização das redes de atenção à saúde de forma integrada regionalmente, compromete este processo $(23,24)$.

O sistema de saúde deve desempenhar suas funções para além do contexto da pandemia, com a expansão de UTI e leitos hospitalares, sendo capaz ainda de reorganizar os fluxos na rede de atendimento a fim de promover a ampliação do acesso e capacidade de resposta às demandas usuais e de urgência e emergência (25). Os resultados sobre a evasão de pacientes apresentados nesse estudo, especialmente em regiões de saúde com pequeno porte populacional, apontam para a necessidade de ampliação na oferta de serviços hospitalares, leitos e profissionais nas diferentes regiões de saúde. A regionalização da saúde, associada às dimensões continentais do Brasil, faz com que muitos pacientes percorram longas distâncias em busca de internação, situação desfavorável em função da pandemia e da rápida evolução da doença (6).

\section{Limitações}

O uso de dados secundários pode estar sujeito a problemas na notificação dos casos. Os dados representam o período de março a outubro de 2020 (antes da segunda onda no Brasil), e é possível que os fluxos de internação ocorram de maneira distinta em outros períodos da pandemia. Também não é possível estimar a partir dos dados a demanda reprimida de internação não realizada, ou seja, um baixo percentual de evasão não necessariamente traduz uma elevada capacidade em atender as demandas da região de saúde. Por fim, não foi possível distinguir os fluxos de internação do setor público e privado, visto que essa distinção não está disponível no banco de dados usado neste estudo. 


\section{Implicação dos resultados para as políticas de saúde}

A análise dos fluxos de internação por COVID-19 nas regiões de saúde pode indicar a necessidade de organização das regiões de saúde para que sejam capazes de cumprir seus objetivos, garantindo o acesso resolutivo à rede de atenção, em tempo oportuno e com qualidade, efetivando o processo de descentralização das ações e serviços de saúde, com o uso racional dos recursos, de modo a reduzir as desigualdades locais e regionais.

\section{Referências}

1. Lurie N, Saville M, Hatchett R, Halton J. Developing Covid-19 Vaccines at Pandemic Speed. N Engl J Med [Internet]. 2020 Mar 30;382(21):1969-73. Available from: https://doi.org/10.1056/NEJMp2005630

2. Sanders JM, Monogue ML, Jodlowski TZ, Cutrell JB. Pharmacologic Treatments for Coronavirus Disease 2019 (COVID-19): A Review. JAMA [Internet]. 2020 May 12;323(18):1824-36. Available from: https://doi.org/10.1001/jama.2020.6019

3. Wilder-Smith A, Freedman DO. Isolation, quarantine, social distancing and community containment: pivotal role for old-style public health measures in the novel coronavirus (2019-nCoV) outbreak. J Travel Med. 2020 Mar;27(2).

4. Wilder-Smith A, Chiew CJ, Lee VJ. Can we contain the COVID-19 outbreak with the same measures as for SARS? Lancet Infect Dis [Internet]. 2020;20(5):e102-7. Available from: http://dx.doi.org/10.1016/S1473-3099(20)30129-8

5. The Lancet Microbe. COVID-19 vaccines: the pandemic will not end overnight. The Lancet Microbe [Internet]. 2021 Jan;2(1):e1. Available from: https://linkinghub.elsevier.com/retrieve/pii/S2666524720302263

6. Noronha KVM de S, Guedes GR, Turra CM, Andrade MV, Botega L, 
Nogueira D, et al. Pandemia por COVID-19 no Brasil: análise da demanda e da oferta de leitos hospitalares e equipamentos de ventilação assistida segundo diferentes cenários. Cad Saude Publica [Internet]. 2020;36(6). Available from:

http://www.scielo.br/scielo.php?script=sci_arttext\&pid=S0102311X2020000605004\&tIng=pt

7. Araújo JL, Oliveira KKD, Freitas RJM. Organização da assistência hospitalar de referência para COVID-19: Relato de experiência. Brazilian J Dev. 2020;6(5):29326-39.

8. FIOCRUZ. Ministério da Saúde. PLANO DE CONTINGÊNCIA DA FIOCRUZ DIANTE DA PANDEMIA DA DOENÇA PELO SARS-CoV-2 (COVID-19) [Internet]. Vol. 1.3. Fiocruz; 2020. Report No.: 1.3. Available from: https://portal.fiocruz.br/documento/plano-de-contingencia-dafiocruz-para-pandemia-de-covid-19-versao-13

9. BRASIL. SRAG 2020 - Banco de Dados de Síndrome Respiratória Aguda Grave - incluindo dados da COVID-19 [Internet]. Ministério da Saúde. openDataSUS. 2020. Available from: https://opendatasus.saude.gov.br/dataset/bd-srag-2020

10. Portela MC, Pereira CC de A, Andrade, Carla LT de, Lima SMaL, Neto FCB, Soares FRG, et al. As regiões de saúde e a capacidade instalada de leitos de UTI e alguns equipamentos para o enfrentamento dos casos graves de Covid-19: Nota Técnica 2 [Internet]. Rio de Janeiro; 2020. Available from: https://www.arca.fiocruz.br/handle/icict/42249

11. Xavier DR, Oliveira RAD de, Barcellos C, Saldanha R de F, Ramalho WM, Laguardia J, et al. As Regiões de Saúde no Brasil segundo internações: método para apoio na regionalização de saúde. Cad Saude Publica [Internet]. 2019;35(suppl 2). Available from: http://www.scielo.br/scielo.php?script=sci_arttext\&pid=S0102$311 \times 2019000805003 \&$ tIng $=p t$

12. Scarpin CT, Steiner MTA, Dias GJC, Steiner Neto PJ. Otimização no serviço de saúde no estado do Paraná: fluxo de pacientes e novas 
configurações hierárquicas. Gestão \& Produção [Internet]. 2008 Aug;15(2):275-90. Available from:

http://www.scielo.br/scielo.php?script=sci_arttext\&pid=S0104$530 \times 2008000200006 \& \operatorname{lng}=p t \& t \operatorname{lng}=p t$

13. Brasil. Ministério da saúde. RESOLUÇÃO No 1, DE 29 DE SETEMBRO DE 2011. Estabelece diretrizes gerais para a instituição de Regiões de Saúde no âmbito do Sistema Único de Saúde (SUS), nos termos do Decreto No 7.508, de 28 de junho de 2011. [Internet]. Brasília: Saúde Legis - Sistema de Legislação da Saúde; 2011. Available from: Saúde Legis - Sistema de Legislação da Saúde

14. Rocha JSY, Monteiro RA, Moreira ML. Hospitalization flow in the public and private systems in the state of Sao Paulo, Brazil. Rev Saude Publica [Internet]. 2015;49. Available from:

http://www.scielo.br/scielo.php?script=sci_arttext\&pid=S0034$89102015000100258 \& \operatorname{lng}=e n \& \operatorname{lng}=$ en

15. Niquini RP, Lana RM, Pacheco AG, Cruz OG, Coelho FC, Carvalho LM, et al. SRAG por COVID-19 no Brasil: descrição e comparação de características demográficas e comorbidades com SRAG por influenza e com a população geral. Cad Saude Publica [Internet]. 2020;36(7):1-12. Available from: https://www.scielo.br/pdf/csp/v36n7/1678-4464-csp-36-07e00149420.pdf

16. Portela MC, Pereira CC de A, Andrade CLT de, Lima SML, Neto FCB, Soares FRG, et al. NOTA TÉCNICA 2: As regiões de saúde e a capacidade instalada de leitos de UTI e alguns equipamentos para o enfrentamento dos casos graves de Covid-19 [Internet]. Rio de Janeiro: Fiocruz; 2020. Available from:

https://portal.fiocruz.br/sites/portal.fiocruz.br/files/documentos/nt_2_portel a_et_al_regioes_de_saude_e_a_capacidade_instalada_de_leitos_de_uti _e_equipamentos_na_covid-19.pdf

17. Viana AL d'Ávila, Bousquat A, Melo GA, Negri Filho A De, Medina MG. Regionalization and Health Networks. Cien Saude Colet. 2018 Jun;23(6):1791-8. 
18. Ribeiro JM, Moreira MR, Ouverney AM, Silva CMFP da. Políticas de saúde e lacunas federativas no Brasil: uma análise da capacidade regional de provisão de serviços. Cien Saude Colet [Internet]. 2017 Apr;22(4):1031-44. Available from:

http://www.scielo.br/scielo.php?script=sci_arttext\&pid=S1413$81232017002401031 \&$ Ing $=p t \&$ tIng $=p t$

19. Brasil. Ministério da saúde. PORTARIA No 95, DE 26 DE JANEIRO DE 2001: NORMA OPERACIONAL DA ASSISTÊNCIA À SAÚDE / SUS NOAS-SUS 01/2001 [Internet]. Brasília: Saúde Legis - Sistema de Legislação da Saúde; 2001. Available from:

http://bvsms.saude.gov.br/bvs/saudelegis/gm/2001/prt0095_26_01_2001. html\#: :text=1 ${ }^{\circ}$ Aprovar\%2C na forma do,Sistema Único de Saúde e

20. Brasil. Ministério da saúde. PORTARIA N 4.279, DE 30 DE DEZEMBRO DE 2010 Estabelece diretrizes para a organização da Rede de Atenção à Saúde no âmbito do Sistema Único de Saúde (SUS). [Internet]. Brasília: Saúde Legis - Sistema de Legislação da Saúde; 2010. Available from: http://bvsms.saude.gov.br/bvs/saudelegis/gm/2010/prt4279_30_12_2010. html

21. Brasil. DECRETO No 7.508, DE 28 DE JUNHO DE 2011. Regulamenta a Lei $n^{\circ} 8.080$, de 19 de setembro de 1990, para dispor sobre a organização do Sistema Único de Saúde - SUS, o planejamento da saúde, a assistência à saúde e a articulação interfederativa, e dá outras $p$ [Internet]. Brasília: Diário Oficial da União; 2011. Available from:

http://www.planalto.gov.br/ccivil_03/_ato20112014/2011/decreto/d7508.htm

22. CONASEMS e CONASS. Guia Orientador para o enfrenta- mento da pandemia Covid-19 na Rede de Atenção à Saúde [Internet]. Brasília; 2020. Available from: https://www.conass.org.br/wpcontent/uploads/2020/05/Instrumento-Orientador-Conass-Conasems.pdf

23. Rabello LEF de S. SUS: o espaço da gestão inovada e dos consensos interfederativos - aspectos jurídicos, administrativos e financeiros. Cien Saude Colet [Internet]. 2009 Jun;14(3):983-983. Available from: 
http://www.scielo.br/scielo.php?script=sci_arttext\&pid=S1413$81232009000300034 \&$ Ing $=p t \&$ tlng=pt

24. Goya N, Andrade LOM de. O Sistema Único de Saúde e o desafio da gestão regionalizada e contratualizada. Rev Bras em Promoção da Saúde [Internet]. 2018 Dec 21;31(4). Available from:

http://periodicos.unifor.br/RBPS/article/view/8773

25. World Health Organization. Operational considerations for case management of COVID-19 in health facility and community: interim guidance, 19 March 2020 [Internet]. Geneva PP - Geneva: World Health Organization; 2020. Available from:

https://apps.who.int/iris/handle/10665/331492 
Tabela 1. Percentual de evasão de residentes da região de saúde para internação por COVID-19, segundo região geográfica, intensidade de demanda por internação e porte populacional da região de saúde de residência do paciente, entre março e outubro de 2020

\begin{tabular}{|c|c|c|c|c|c|c|c|c|}
\hline \multirow[b]{2}{*}{ Região } & \multirow{2}{*}{$\begin{array}{l}\text { Período de } \\
\text { Demanda por } \\
\text { Internação }\end{array}$} & \multicolumn{7}{|c|}{ Porte Populacional da Região de Saúde de Residência do Paciente Internado } \\
\hline & & $\begin{array}{c}\text { até } 100 \\
\text { mil }\end{array}$ & $\begin{array}{c}100 \text { a } 200 \\
\text { mil }\end{array}$ & $\begin{array}{c}200 \text { a } 500 \\
\text { mil }\end{array}$ & $\begin{array}{c}500 \text { mil a } 1 \\
\text { milhão }\end{array}$ & $\begin{array}{c}1 \text { a } 2 \\
\text { milhões }\end{array}$ & $\begin{array}{c}\text { Acima de } 2 \\
\text { milhões }\end{array}$ & Total \\
\hline \multirow{3}{*}{ Norte } & Total & $38.5 \%$ & $24.8 \%$ & $15.9 \%$ & $9.6 \%$ & - & $1.7 \%$ & $9.8 \%$ \\
\hline & Maior Demanda & $45.1 \%$ & $27.0 \%$ & $15.9 \%$ & $11.4 \%$ & - & $1.8 \%$ & $10.3 \%$ \\
\hline & Menor Demanda & $36.3 \%$ & $23.9 \%$ & $15.9 \%$ & $8.9 \%$ & - & $1.7 \%$ & $9.6 \%$ \\
\hline \multirow{3}{*}{ Nordeste } & Total & $62.9 \%$ & $55.7 \%$ & $40.7 \%$ & $23.6 \%$ & $5.0 \%$ & $0.8 \%$ & $17.6 \%$ \\
\hline & Maior Demanda & $61.9 \%$ & $55.9 \%$ & $42.5 \%$ & $24.7 \%$ & $4.8 \%$ & $0.9 \%$ & $19.1 \%$ \\
\hline & Menor Demanda & $63.5 \%$ & $55.6 \%$ & $40.0 \%$ & $23.3 \%$ & $5.0 \%$ & $0.8 \%$ & $17.0 \%$ \\
\hline \multirow{3}{*}{ Sudeste } & Total & $34.8 \%$ & $20.4 \%$ & $11.2 \%$ & $9.9 \%$ & $23.3 \%$ & $7.5 \%$ & $10.6 \%$ \\
\hline & Maior Demanda & $37.2 \%$ & $19.3 \%$ & $12.0 \%$ & $9.4 \%$ & $22.3 \%$ & $7.0 \%$ & $10.5 \%$ \\
\hline & Menor Demanda & $33.8 \%$ & $20.8 \%$ & $11.0 \%$ & $10.0 \%$ & $23.5 \%$ & $7.6 \%$ & $10.7 \%$ \\
\hline \multirow{3}{*}{ Sul } & Total & - & $23.9 \%$ & $14.1 \%$ & $8.9 \%$ & $4.2 \%$ & $1.3 \%$ & $8.8 \%$ \\
\hline & Maior Demanda & - & $24.7 \%$ & $15.1 \%$ & $9.1 \%$ & $4.1 \%$ & $1.2 \%$ & $10.3 \%$ \\
\hline & Menor Demanda & - & $23.6 \%$ & $13.7 \%$ & $8.8 \%$ & $4.2 \%$ & $1.3 \%$ & $8.4 \%$ \\
\hline \multirow{3}{*}{$\begin{array}{l}\text { Centro- } \\
\text { Oeste }\end{array}$} & Total & $36.8 \%$ & $28.8 \%$ & $19.1 \%$ & $28.9 \%$ & $7.5 \%$ & $1.0 \%$ & $12.4 \%$ \\
\hline & Maior Demanda & $39.5 \%$ & $32.5 \%$ & $17.7 \%$ & $26.2 \%$ & $8.5 \%$ & $1.2 \%$ & $14.2 \%$ \\
\hline & Menor Demanda & $35.6 \%$ & $27.4 \%$ & $19.6 \%$ & $29.6 \%$ & $7.3 \%$ & $1.0 \%$ & $11.9 \%$ \\
\hline \multirow{3}{*}{ Brasil } & Total & $36.9 \%$ & $30.9 \%$ & $20.3 \%$ & $14.0 \%$ & $12.8 \%$ & $5.2 \%$ & $11.9 \%$ \\
\hline & Maior Demanda & $40.0 \%$ & $32.1 \%$ & $22.2 \%$ & $14.7 \%$ & $11.3 \%$ & $4.7 \%$ & $12.9 \%$ \\
\hline & Menor Demanda & $35.5 \%$ & $30.4 \%$ & $19.7 \%$ & $13.8 \%$ & $13.2 \%$ & $5.3 \%$ & $11.7 \%$ \\
\hline
\end{tabular}

Quadro 1. Municípios do interior a que figuraram como destino mais frequente dos fluxos migratórios de usuários com COVID-19 para mais de um município, de março a outubro de 2020.

\begin{tabular}{|c|c|c|c|c|c|}
\hline Região & UF & Município & População $^{\text {b }}$ & Macroc $^{c}$ & IDH \\
\hline \multirow[t]{3}{*}{ Norte } & $\mathrm{RO}$ & Cacoal & 85.893 & Não & 0,718 \\
\hline & $\mathrm{AC}$ & Cruzeiro do Sul & 89.072 & Não & 0,664 \\
\hline & PA & Santarém & 306.480 & Sim & 0,691 \\
\hline \multirow[t]{2}{*}{ Centro-Oeste } & $\mathrm{GO}$ & Anápolis & 391.772 & Sim & 0,737 \\
\hline & $\mathrm{GO}$ & Águas Lindas de Goias & 217.698 & Sim & 0,686 \\
\hline \multirow[t]{15}{*}{ Nordeste } & MA & Imperatriz & 259.337 & Sim & 0,731 \\
\hline & $\mathrm{PI}$ & Parnaíba & 153.482 & Sim & 0,687 \\
\hline & CE & Sobral & 210.711 & Sim & 0,714 \\
\hline & $\mathrm{CE}$ & Juazeiro do Norte & 276.264 & Sim & 0,694 \\
\hline & $\mathrm{RN}$ & Mossoró & 300.618 & Sim & 0,720 \\
\hline & PB & Campina Grande & 411.807 & Sim & 0,720 \\
\hline & PB & Patos & 108.192 & Sim & 0,701 \\
\hline & PB & Cajazeiras & 62.289 & Não & 0,679 \\
\hline & $\mathrm{PE}$ & Petrolina & 354.317 & Sim & 0,697 \\
\hline & $\mathrm{PE}$ & Serra Talhada & 86.915 & Sim & 0,661 \\
\hline & $\mathrm{AL}$ & Arapiraca & 233.047 & Sim & 0,649 \\
\hline & $\mathrm{BA}$ & Vitória da Conquista & 341.128 & Sim & 0,678 \\
\hline & $\mathrm{BA}$ & Porto Seguro & 150.658 & Não & 0,676 \\
\hline & $\mathrm{BA}$ & Itabuna & 213.685 & Sim & 0,712 \\
\hline & $\mathrm{BA}$ & Barreiras & 156.975 & Sim & 0,721 \\
\hline \multirow[t]{4}{*}{ Sudeste } & MG & Montes Claros & 413.487 & Sim & 0,770 \\
\hline & $\mathrm{MG}$ & Teófilo Otoni & 140.937 & Sim & 0,701 \\
\hline & MG & Governador Valadares & 281.046 & Sim & 0,727 \\
\hline & MG & Ipatinga & 265.409 & Sim & 0,771 \\
\hline
\end{tabular}




\begin{tabular}{|l|c|l|c|c|c|}
\hline & MG & Uberaba & 337.092 & Sim & 0,772 \\
\hline & MG & Divinópolis, & 240.408 & Sim & 0,764 \\
\hline & MG & Patos de Minas & 153.585 & Sim & 0,765 \\
\hline & MG & Passos & 115.337 & Não & 0,756 \\
\hline & MG & Poços de Caldas & 168.641 & Sim & 0,779 \\
\hline & MG & Varginha & 136.602 & Não & 0,778 \\
\hline & MG & Pouso Alegre & 152.549 & Não & 0,774 \\
\hline & MG & Muriaé & 109.392 & Não & 0,734 \\
\hline & MG & Juiz de Fora & 573.285 & Sim & 0,778 \\
\hline & SP & São José do Rio Preto & 464.983 & Sim & 0,797 \\
\hline & SP & Presidente Prudente & 230.371 & Sim & 0,806 \\
\hline & SP & Araçatuba & 464.983 & Não & 0,788 \\
\hline & SP & Ribeirão Preto & 711.825 & Sim & 0,800 \\
\hline & SP & Araraquara & 238.339 & Não & 0,815 \\
\hline & SP & Bauru & 379.297 & Sim & 0,801 \\
\hline & SP & Botucatu & 148.130 & Não & 0,800 \\
\hline & SP & Piracicaba & 407.252 & Sim & 0,785 \\
\hline & SP & Campinas & 1.213 .792 & Sim & 0,805 \\
\hline & SP & São José dos Campos & 729.737 & Sim & 0,807 \\
\hline & PR & Londrina & 575.377 & Sim & 0,778 \\
\hline & PR & Maringá & 430.157 & Sim & 0,808 \\
\hline & PR & Ponta Grossa & 355.336 & Não & 0,763 \\
\hline & SC & Chapecó & 224.013 & Sim & 0,790 \\
\hline & SC & Itajaí & 223.112 & Sim & 0,795 \\
\hline & RS & Caxias do Sul & 517.451 & Sim & 0,782 \\
\hline & RS & Passo Fundo & 204.722 & Sim & 0,776 \\
\hline & RS & Santa Rosa & 283.575 & Não & 0,769 \\
\hline & RS & Santa Maria & Sim & 0,784 \\
\hline
\end{tabular}

a Municípios diferentes das capitais estaduais, assim como daqueles que compõem suas regiões metropolitanas; ${ }^{\mathrm{b}}$ Estimativas da população residente em 2020 nos municípios brasileiros - data de referência: 01/07/2020 (IBGE);

`Sim - se é o município mais populoso da sua macrorregião de saúde.
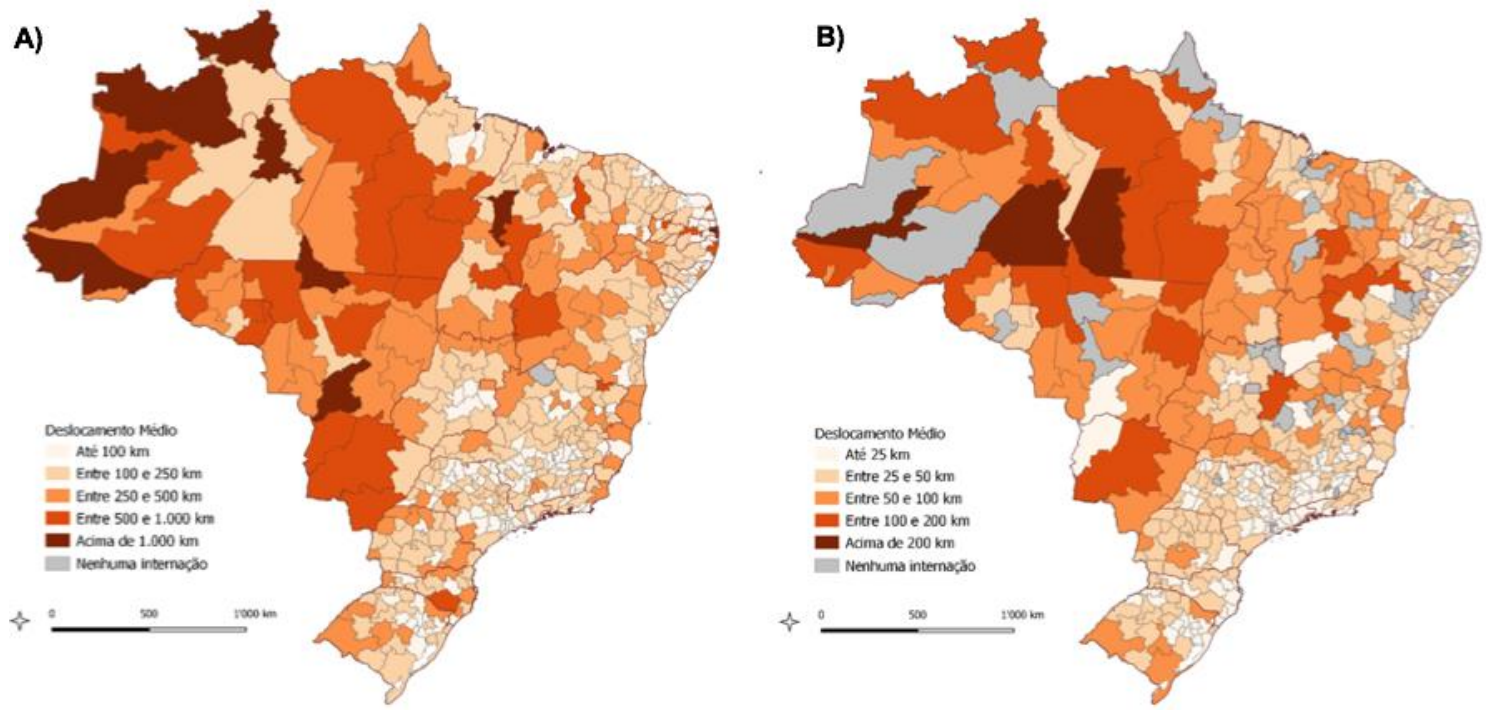

Figura 1. Distância média de deslocamento para internação por COVID-19 realizadas fora da região de saúde $(A)$ e dentro de cada região de saúde $(B)$ no Brasil, de março a outubro de 2020 

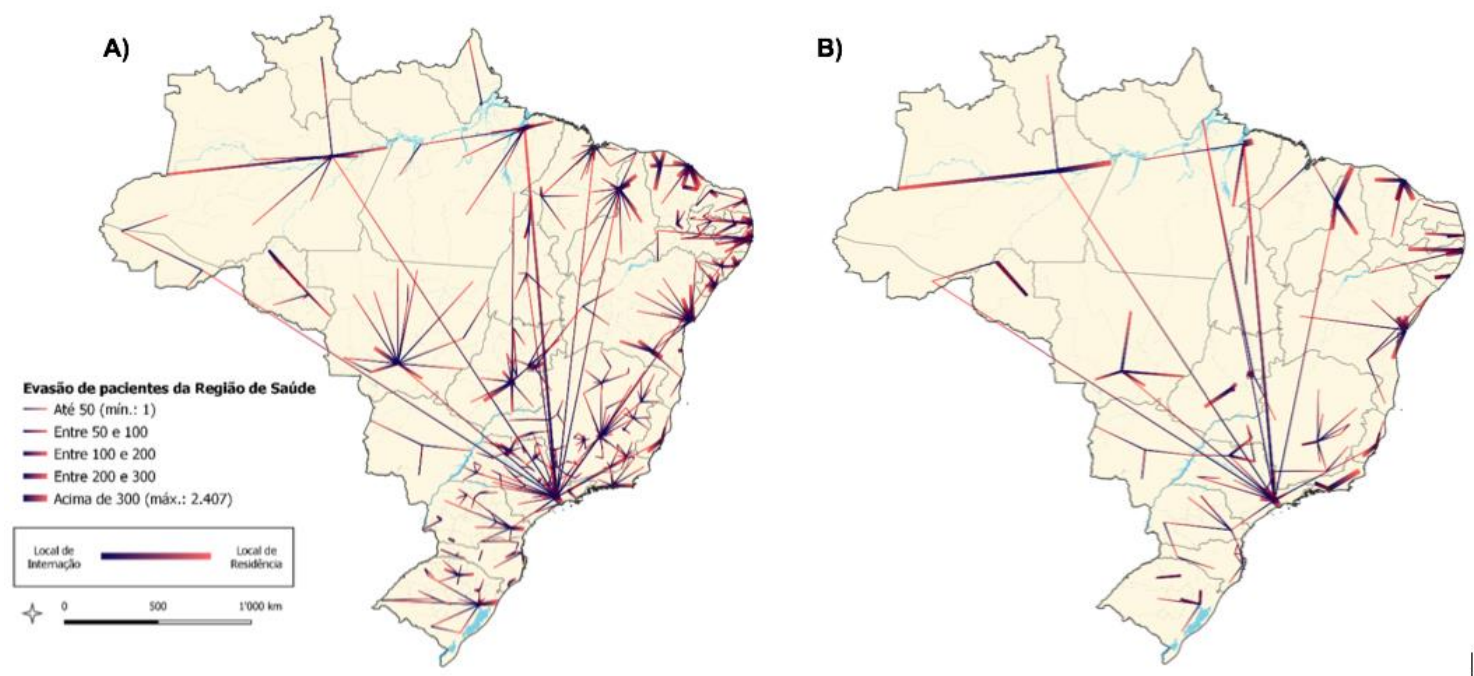

Figura 2. Fluxo mais intenso de evasão de pacientes para internação por COVID-19 entre as regiões de saúde (A) e as macrorregiões de saúde (B) brasileiras, de março a outubro de 2020

Nota: As coordenadas utilizadas como origem e destino para representar os fluxos entre as macrorregiões de saúde foram definidas a partir da coordenada da sede do município mais populoso da macrorregião.

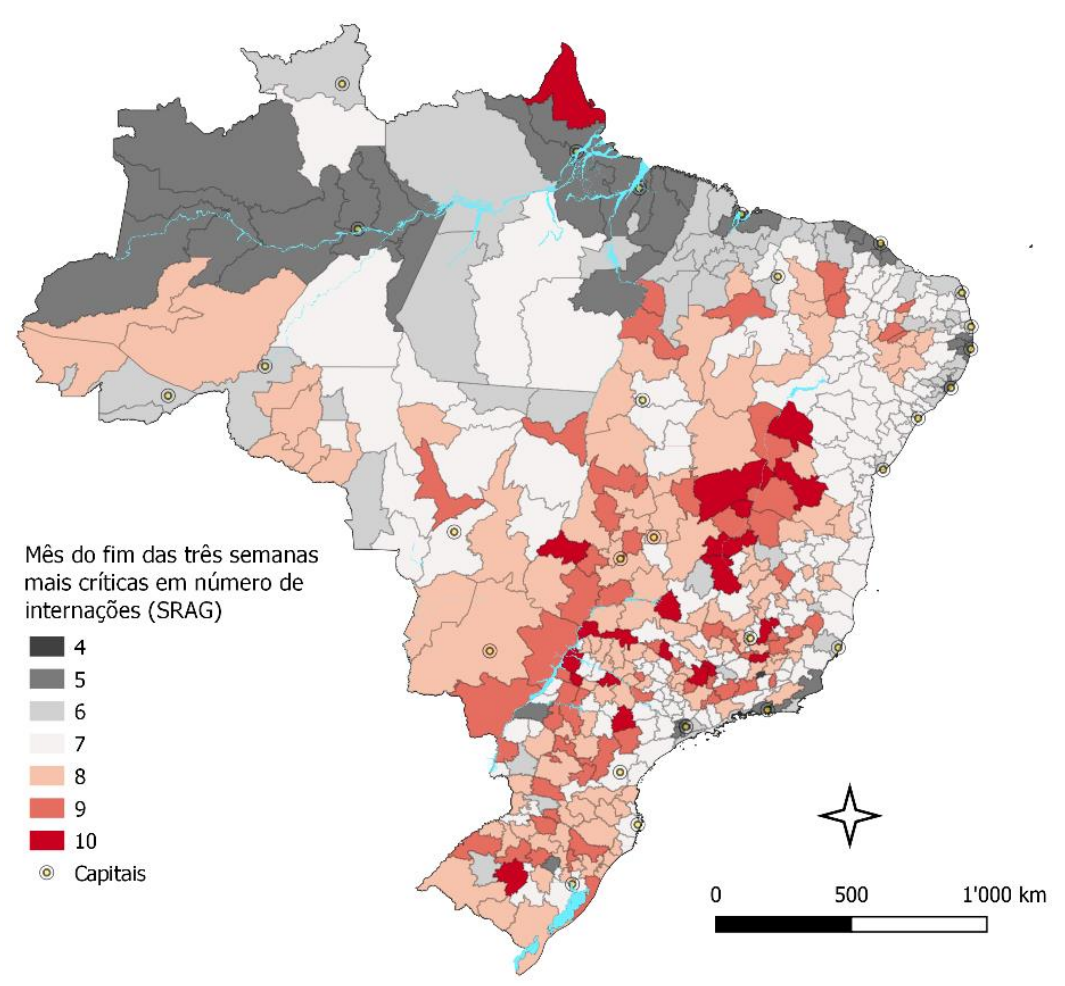

Figura 3. Temporalidade de período crítico de internação por COVID-19 no Brasil, de acordo com a região de saúde, entre março e outubro de 2020 

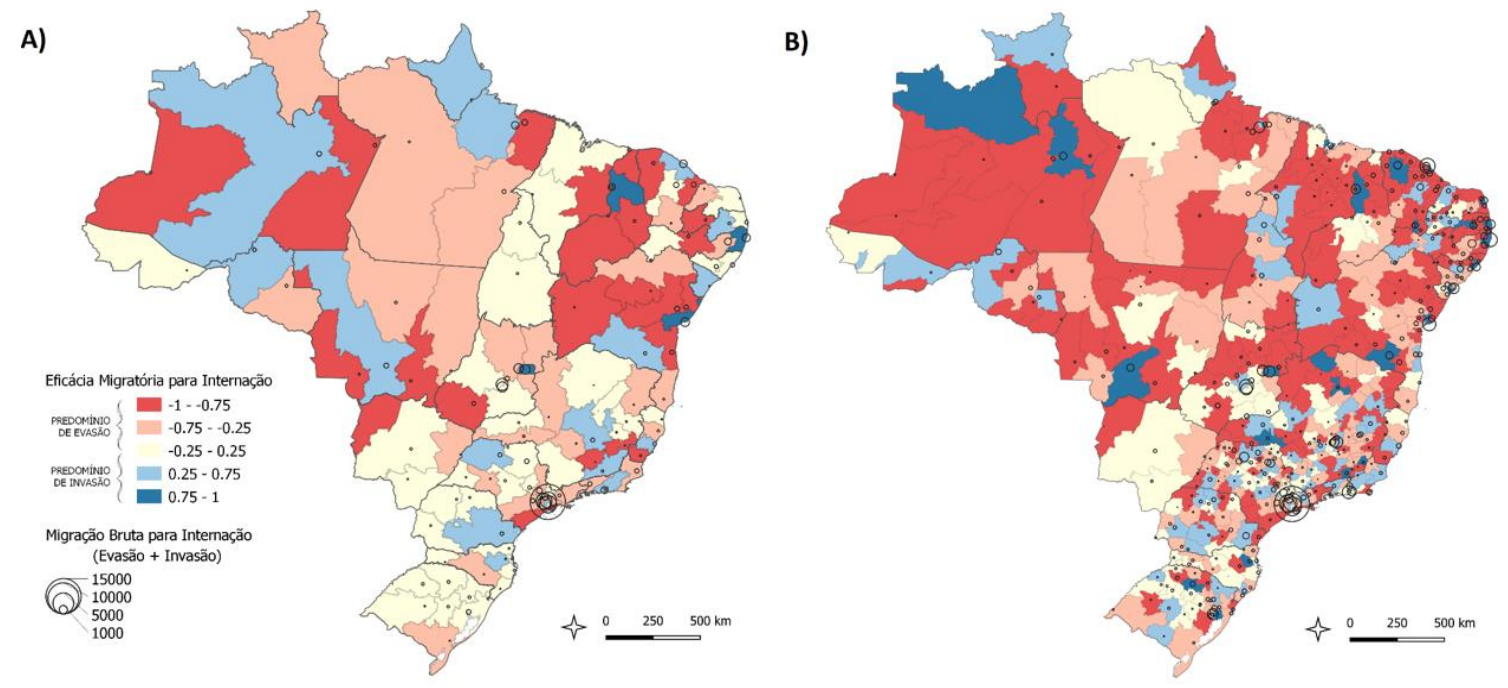

Figura 4. Eficácia migratória para internação por COVID-19 no Brasil por macrorregião de saúde (A) e por região de saúde (B), entre março e outubro de 2020 